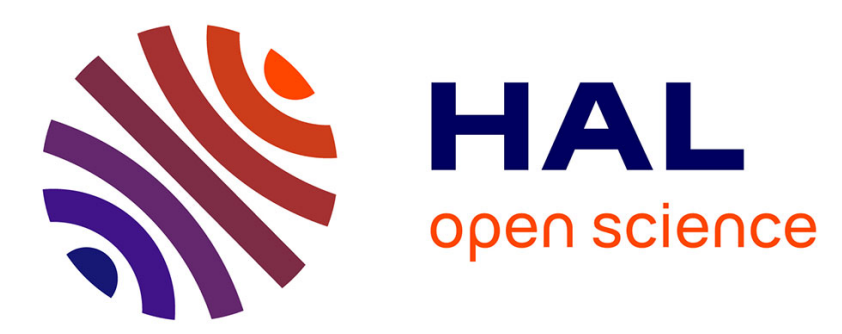

\title{
Parametric Replay-Based Simulation of Underwater Acoustic Communication Channels
}

\author{
François-Xavier Socheleau, Christophe Laot, Jean-Michel Passerieux
}

\section{To cite this version:}

François-Xavier Socheleau, Christophe Laot, Jean-Michel Passerieux. Parametric Replay-Based Simulation of Underwater Acoustic Communication Channels. IEEE Journal of Oceanic Engineering, 2015, 40 (4), pp.796 - 806. 10.1109/JOE.2015.2458211 . hal-01224555

\section{HAL Id: hal-01224555 \\ https://hal.science/hal-01224555}

Submitted on 11 Jun 2021

HAL is a multi-disciplinary open access archive for the deposit and dissemination of scientific research documents, whether they are published or not. The documents may come from teaching and research institutions in France or abroad, or from public or private research centers.
L'archive ouverte pluridisciplinaire HAL, est destinée au dépôt et à la diffusion de documents scientifiques de niveau recherche, publiés ou non, émanant des établissements d'enseignement et de recherche français ou étrangers, des laboratoires publics ou privés. 


\title{
Parametric Replay-Based Simulation of
}

\section{Underwater Acoustic Communication Channels}

\author{
François-Xavier Socheleau, Christophe Laot, Jean-Michel Passerieux
}

\begin{abstract}
This paper presents an underwater acoustic channel simulation methodology that combines parametric modeling with stochastic replay of at-sea measured channel impulse responses. The motivation behind this approach is to extend the scope of use of replay-based methods by allowing some parameterization of the channel properties while complying with some level of realism. Such an approach is beneficial for extensive testing of communication links. Based on a relative entropy minimization between the original time-varying channel impulse response and the simulated one, the idea is to deliberately distort the original channel statistics in order to meet some user-specified constraints. A particular attention is given to constraints on the channel Doppler spread and on the level of covariance between channel taps. The testing capabilities provided by parametric replay-based simulations are illustrated with real data collected in the bay of Brest (France).
\end{abstract}

\section{Index Terms}

Underwater acoustic communications, channel simulation, stochastic replay, relative entropy minimization

\section{INTRODUCTION}

The use of underwater acoustic channel simulators appears as an efficient way of limiting the design and test efforts of communication systems when at-sea experimentation is costly and difficult to

F.-X. Socheleau and C. Laot are with Institut Mines-Telecom, Telecom Bretagne, UMR CNRS 6285 Lab-STICC, France (e-mail: $\{$ fx.socheleau, christophe.laot $\} @$ telecom-bretagne.eu)

J.-M. Passerieux is with Thales Underwater Systems, France (e-mail: jean-michel.passerieux@ fr.thalesgroup.com).

A preliminary version of this paper has been presented at the Conference on Underwater Communications and Networking (UComms) held in Sestri Levante, Italy, in September 2014 [1]. 
conduct. Among the various simulation strategies proposed in the literature, replay of time-varying impulse responses (TVIRs) measured in situ has emerged as a relevant and accurate underwater acoustic communication (UAC) channel simulation method. As described in [2]-[5], replay-based simulators either strictly reproduce the measured TVIR in laboratory (a.k.a. direct replay), or generate new random TVIRs with statistical properties identical to the original measurement (a.k.a. stochastic replay). ${ }^{1}$ From a single measurement, it is thus possible to compare competing transmission schemes when faced with the same realistic environment [5]. Thanks to Monte-Carlo simulations, design and validation metrics such as bit error rate [3]-[5], capacity bounds [6]-[8] or fading statistics [4] can be computed with a good accuracy. Replay-based methods are intrinsically realistic and do not require physical input parameters for which numerical values may not be easy to set. However, unlike parametric model-based simulators such as [9]-[15], the diversity of environments simulated by replay-based methods can be very limited. It actually depends on the number of sea-probed TVIR available.

The best way to increase diversity with replay-based simulation is to collect a large amount of data in different locations at different times. Obviously, this is the best option only if large-scale experiments can be conducted. Another approach that is put forward in this paper is to create artificial diversity in some statistical sense. Such an approach does not consider diversity in terms of channel measurement conditions (e.g. sea state or deployment geometry) but rather in terms of statistical moments of random TVIRs. This strategy can be relevant for testing the robustness of underwater acoustic communication (UAC) systems. In fact, systems designed on the basis of a statistical channel model can be expected to work in a variety of different propagation environments. Existing replay-based methods can already create some artificial diversity by exploiting their ability to separate first-order statistics of the measured impulse response from second-order statistics. A first example was given in [16] where the power ratios between the specular and the scattered components of the channel were artificially modified to test the receiver's robustness to random scattering. To the best of our knowledge, this is the only attempt made so far to purposely distort the channel statistics in the context of stochastic replay.

In this work, we extend the idea of [16] and seek to build a replay-based simulation strategy that allows some level of parameterization in order to enlarge its scope of use. The problem we want to tackle is the following: given prior information on UAC channels that is available through a measured impulse

\footnotetext{
${ }^{1}$ Note that stochastic replay does not apply to all kind of channels in its current implementation. Cyclostationary channels or multi-scale multi-lag channels are typical examples that fall outside the domain of applicability of such a method (ref. to [5] for more details).
} 
response, can we build a channel model that satisfies user-specified constraints while being "as close as possible" to the original TVIR? Constraining the model means that we want to control some of its statistical properties through specific parameters. In this paper, a specific focus is placed on the control of second order statistics such as the Doppler spread and the covariance level between taps. "Being as close as possible" is a way to achieve some level of realism as provided by the original TVIR. As shown in Section III, this problem can be addressed by optimizing an information-theoretic criterion that formally defines the concept of model proximity.

The paper is organized as follows: Section II is devoted to the presentation of the replay-based channel simulation strategy which our framework is based on. Section III presents the method used to deliberately distort the original TVIR statistics in order to meet the desired constraints. Numerical examples of the proposed channel simulation strategy applied to real data are provided in Section IV. Finally, conclusions and perspectives are discussed in Section V.

Notations: Throughout this paper, uppercase boldface letters denote matrices, e.g., A. The superscripts $T$ and ${ }^{\dagger}$ denote transposition and Hermitian transposition, respectively. The entries of a matrix $\mathbf{A}$ are denoted by $[\mathbf{A}]_{k, n}$ and $\operatorname{tr}[\cdot]$ denotes the trace. $\Re$ and $\Im$ denote real and imaginary part, respectively. Finally, $\mathbb{E}\{$.$\} denotes expectation.$

\section{REPLAY-BASED CHANNEL MODEL}

We consider a doubly selective underwater acoustic channel, modeled as a random linear time-varying system $\mathbb{H}$ that maps input signals $x(t)$ onto output signals $z(t)$ according to the I/O relationship

$$
z(t)=(\mathbb{H} x)(t)+w(t)=\int_{\tau} h_{\mathbb{H}}(\tau, t) x(t-\tau) d \tau+w(t),
$$

where $h_{\mathbb{H}}(\tau, t)$ is the channel impulse response and $w(t)$ denotes the ambient noise. As common practice in replay-based simulation, the input of the simulator is a discrete-time baseband estimate of the in-situ channel impulse response whose mean Doppler shift has been removed [3]-[5]. Such an estimate is denoted as $h_{l}(k)$ where $l \in\{0, \cdots, L-1\}$ is the tap index and $k \in\{0, \cdots, K-1\}$ is the time index. Channel sounding is classically performed by transmitting a pseudonoise probe signal or a chirp signal that is passed through a matched-filter at reception [17]. A common alternative method is to combine a phase-shift keyed symbol stream with the least mean squares estimator (LMS) [4]. Note that the removal of the mean Doppler shift is required to mitigate the drift of multipath arrivals that could obscure the estimate of the taps statistics. Once the channel statistical properties have been estimated, this mean Doppler shift can be reinserted at the output of the simulator by performing time resampling. 
In agreement with [4], $\left\{h(k) \triangleq\left[h_{0}(k), h_{1}(k), \cdots, h_{L-1}(k)\right]^{T}, k \in \mathbb{Z}\right\}$ is modeled as a multi-variate trend stationary random process so that, for all $k, k_{1}$ and $k_{2} \in \mathbb{Z}$

$$
h_{l}(k)=\bar{h}_{l}(k)+\tilde{h}_{l}(k),
$$

with

$$
\mathbb{E}\left\{h_{l}(k)\right\}=\bar{h}_{l}(k),
$$

and

$$
\begin{aligned}
& \mathbb{E}\left\{\tilde{h}_{l}\left(k_{1}\right) \tilde{h}_{p}^{*}\left(k_{2}\right)\right\}=\mathbb{E}\left\{\tilde{h}_{l}(k) \tilde{h}_{p}^{*}\left(k+k_{2}-k_{1}\right)\right\}, \\
& \mathbb{E}\left\{\tilde{h}_{l}\left(k_{1}\right) \tilde{h}_{p}\left(k_{2}\right)\right\}=\mathbb{E}\left\{\tilde{h}_{l}(k) \tilde{h}_{p}\left(k+k_{2}-k_{1}\right)\right\} .
\end{aligned}
$$

$\bar{h}_{l}(k)$ is called the trend and is a slowly time-varying deterministic component. ${ }^{2} \tilde{h}_{l}(k)$ is a zero-mean wide-sense stationary random process assumed to be Gaussian. This model describes the UAC channel as a multivariate Rician fading process with a slowly time-varying mean. $\bar{h}_{l}(k)$ can be interpreted as the contribution of (pseudo) deterministic physical phenomena to channel fluctuations, i.e. $\bar{h}_{l}(k)$ is a (pseudo) specular component, and $\tilde{h}_{l}(k)$ represents the channel fluctuations attributable to scatterers that result in fast fading. Note that since no particular assumption is made about the correlation of scatterers, the model is very general and includes the wide-sense stationary uncorrelated scattering (WSSUS) model as a subset.

Based on (2), standard replay methods first consist in isolating both components $\bar{h}_{l}(k)$ and $\tilde{h}_{l}(k)$ from $h_{l}(k)$. This can be done by estimating the specular component $\bar{h}_{l}(k)$ either by simple time averaging when $\bar{h}_{l}(k)$ is (approximately) time-invariant [3], [5], [18] or by an empirical mode decomposition [4] in the general time-varying case. $\tilde{h}_{l}(k)$ is then obtained as the difference between $h_{l}(k)$ and $\bar{h}_{l}(k)$. An example of the channel decomposition is shown in Fig. 1.

Finally, stochastic replay consists in drawing new realizations of the channel random component based on the observation $\tilde{h}_{l}(k)$. These realizations are then added to the original specular component $\bar{h}_{l}(k)$ to obtain a new TVIR. New realizations of process $\left\{\tilde{h}(k) \triangleq\left[\tilde{h}_{0}(k), \tilde{h}_{1}(k), \cdots, \tilde{h}_{L-1}(k)\right]^{T}\right\}$ can be obtained either by explicit estimation of its second order statistics, as given by the scattering function for instance [3], and by then filtering white Gaussian noises, or they can be generated simply by adding noise on the phase of the discrete Fourier transform of the observation $\tilde{h}_{l}(k)$ [4], [19].

\footnotetext{
${ }^{2}$ Note that $\bar{h}_{l}(k)$ can be null for some $l$.
} 


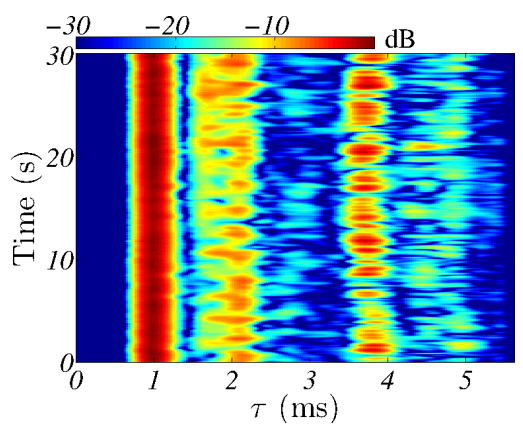

(a)

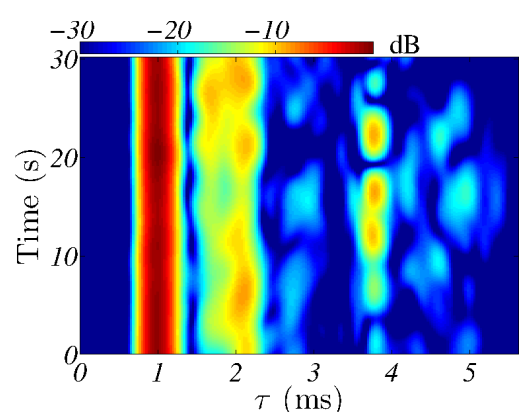

(b)

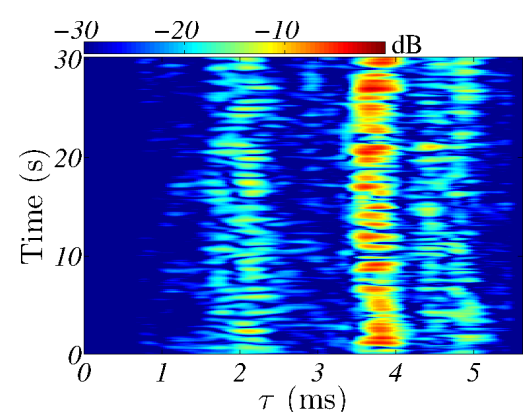

(c)

Fig. 1. Example of TVIR decomposition using EMD. (a) Magnitude of a TVIR measured in situ, i.e., $\left|h_{l}(k)\right|$, (b) Magnitude of the trend, i.e., $\left|\bar{h}_{l}(k)\right|$, (c) Magnitude of the random component, i.e., $\left|\tilde{h}_{l}(k)\right|$. This TVIR has been extracted from an experiment conducted in 2007 in the Bay of Brest (see Sec. IV for more details on the experiment) and estimated using a data-aided LMS algorithm.

In this work, we consider the first approach and assume that we are able to estimate the full secondorder statistics of $\{\tilde{h}(k)\}$ given by the $2 L \times 2 L$ cross-spectral density matrix expressed as

$$
\mathbf{S}_{\tilde{h}}(\omega) \triangleq\left[\begin{array}{ll}
\mathbf{S}_{\tilde{h}}^{\Re, \Re}(\omega) & \mathbf{S}_{\tilde{h}}^{\Re, \Im}(\omega) \\
\mathbf{S}_{\tilde{h}}^{\Im, \Re}(\omega) & \mathbf{S}_{\tilde{h}}^{\Im, \Im}(\omega)
\end{array}\right],
$$

where the matrix sub-block $\mathbf{S}_{\tilde{h}}^{\Re, \Im}(\omega)$ of size $L \times L$ satisfies for all $\omega \in[-\pi, \pi]$

$$
\left[\mathbf{S}_{\tilde{h}}^{\Re, \Im}(\omega)\right]_{l p}=\sum_{u=-\infty}^{+\infty} \mathbb{E}\left\{\Re\left\{\tilde{h}_{l}(k)\right\} \Im\left\{\tilde{h}_{p}(k+u)\right\}\right\} e^{-i u \omega} .
$$

The matrix blocks $\mathbf{S}_{\tilde{h}}^{\Re, \Re}(\omega), \mathbf{S}_{\tilde{h}}^{\Im, \Re}(\omega), \mathbf{S}_{\tilde{h}}^{\Im, \Im}(\omega)$ are defined analogously to (6). Note that the assumption of taps with independent real and imaginary parts combined with the common assumption of uncorrelated scattering lead to a diagonal matrix $\mathbf{S}_{\tilde{h}}(\omega)$. This is not the case here since we take into account the possible covariance between channel taps as well as the possible covariance between the real and the imaginary part of a single tap. The latter covariance is typically observed when the scattering is nonisotropic [20], [21]. The auto-spectral density functions, i.e. the diagonal elements of $\mathbf{S}_{\tilde{h}}(\omega)$, are real and nonnegative functions of $\omega$ and the cross-spectral density functions, i.e. the non-diagonal elements of $\mathbf{S}_{\tilde{h}}(\omega)$, are generally complex functions of $\omega$. In addition, matrix $\mathbf{S}_{\tilde{h}}(\omega)$ is positive definite and satisfies the following relations

$$
\begin{aligned}
\mathbf{S}_{\tilde{h}}(\omega) & =\mathbf{S}_{\tilde{h}}^{\dagger}(\omega), \\
{\left[\mathbf{S}_{\tilde{h}}(\omega)\right]_{l p} } & =\left[\mathbf{S}_{\tilde{h}}^{*}(-\omega)\right]_{l p} .
\end{aligned}
$$




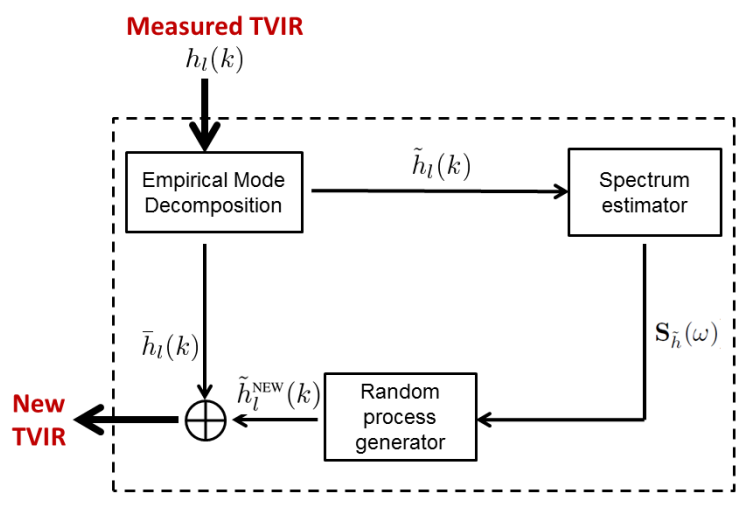

(a)

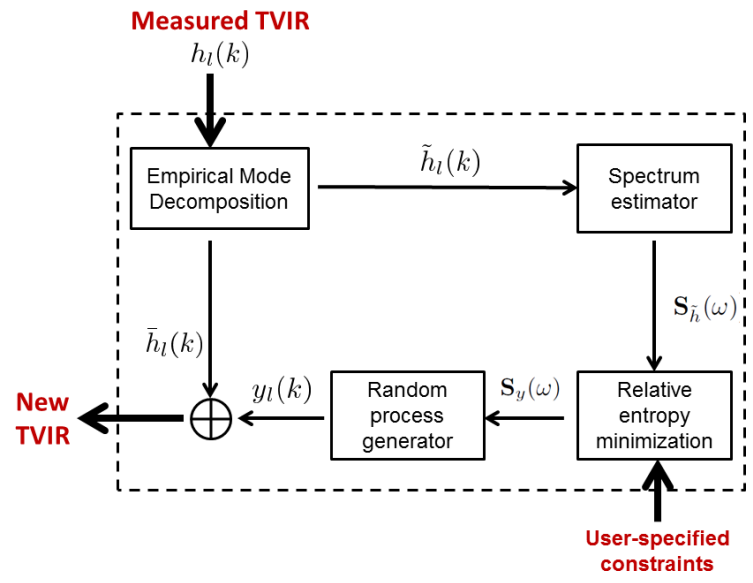

(b)

Fig. 2. Block diagram of replay-based methods, a) standard replay-based simulator as described in Sec.II, b) parametric replay-based simulator.

Estimation of $\mathbf{S}_{\tilde{h}}(\omega)$ can be performed with common spectral estimation methods (Welch, Burg, Correlogram, etc.) as long as the duration of the sea-probed TVIR is much greater than the coherence time of each process $\left\{\tilde{h}_{l}(k)\right\}_{k}$. In most experiments, this duration is on the order of several tens to several hundreds of seconds, which is large in comparison to the coherence time of most UAC channels that is usually lower than few hundreds of milliseconds [17].

Based on the spectral representation (5), it is then possible to draw new realizations of process $\{\tilde{h}(k)\}$ using existing techniques for simulating multivariate stationary Gaussian ergodic processes such as those presented in [22]-[24]. A general overview of such methods is presented in Appendix A. The overall standard replay-based simulation methodology is illustrated in Figure 2-(a).

\section{PARAMETERIZATION OF REPLAY-BASED SIMULATION}

\section{A. General methodology and theoretical background}

As discussed in the introduction, the main drawback of stochastic replay lies in its lack of diversity or flexibility. In this section, we show how it is possible to extend the scope of use of such replaybased modeling strategy by allowing some degree of parameterization. More specifically, the proposed simulation framework consists in generating realizations of a new multivariate Gaussian random process that satisfies some prescribed constraints, while being "as close as possible" to the reference process 
$\left\{\tilde{h}_{l}(k)\right\}$. For instance, considering the second-order statistics only, we show how to specify constraints on the Doppler spread in order to know the ability of a receiver to track the fluctuations of a channel. Similarly, analyzing the impact of correlated scattering on the demodulation performance can be of great interest. Since a realistic prior information is available through the matrix $\mathbf{S}_{\tilde{h}}(\omega)$, it is rather natural to exploit this knowledge to build the new model.

The concept of model proximity can be formally defined through the relative entropy rate between processes, which, as shown in this section, is also a pseudo-distance between spectral density matrices for Gaussian processes. Building models with entropy-based criteria is a common procedure in statistical inference [25]-[31]. Such an approach is justified on the basis of avoiding the arbitrary introduction of unknown information. ${ }^{3}$ Note that entropy-based models are not meant to represent the physical reality of the channel but rather a state of knowledge on this reality. In our case, this state of knowledge is provided by the sea-probed TVIR as well as the user-specified constraints. For Gaussian processes, the relative entropy is defined as follows.

Let $\left\{y(k) \triangleq\left[y_{1}(k), y_{2}(k), \cdots, y_{M}(k)\right]^{T}, k \in \mathbb{Z}\right\}$ be a Gaussian random process taking values in $\mathbb{R}^{M}$ and let $p_{\mathbf{Y}_{[-n, n]}}$ denote the joint probability density function of $\mathbf{Y}_{[-n, n]}=[y(-n), y(-n+1), \cdots, y(n-$ $1), y(n)]$, the differential entropy rate of $y$ is defined as

$$
\mathrm{h}_{r}(y)=\lim _{n \rightarrow+\infty} \frac{1}{2 n+1} H\left(p_{\mathbf{Y}_{[-n, n]}}\right),
$$

where $H(\cdot)$ denotes the differential entropy [32]. Entropy rates can be considered as a tool for quantitative characterization of dynamic processes evolving in time. For multivariate Gaussian processes, it can be seen as a single metric that carries all the information on the second-order statistics (time fluctuations as well as covariance across individual processes). If $\mathbf{S}_{y}(\omega)$ denotes the cross-spectral density matrix of $y$, then it can be shown [32] that

$$
\mathrm{h}_{r}(y)=\frac{M}{2} \log (2 \pi e)+\frac{1}{4 \pi} \int_{-\pi}^{\pi} \log \operatorname{det} \mathbf{S}_{y}(\omega) d \omega .
$$

The concept of entropy rate can be extended as a metric for comparing two random processes. Let $\{y(k), k \in \mathbb{Z}\}$ and $\{z(k), k \in \mathbb{Z}\}$ be two random processes, the relative entropy rate between $y$ and $z$ is defined as

$$
D_{r}(y \| z)=\lim _{n \rightarrow+\infty} \frac{1}{2 n+1} D\left(p_{\mathbf{Y}_{[-n, n]}} \| p_{\mathbf{Z}_{[-n, n]}}\right)
$$

\footnotetext{
${ }^{3}$ It is shown in [26] that the principle of minimum relative entropy is the only method of inductive inference that leads "to consistent results where there are different ways of taking the same information into account".
} 
where $D(\cdot \| \cdot)$ denotes the relative entropy [32]. If $y$ and $z$ are jointly Gaussian with cross-spectral density matrices $\mathbf{S}_{y}(\omega)$ and $\mathbf{S}_{z}(\omega)$, respectively, then, under some regularity conditions (see [30] for details), their relative entropy satisfies

$$
D_{r}(y \| z)=\frac{1}{4 \pi} \int_{-\pi}^{\pi}\left(\log \operatorname{det}\left(\mathbf{S}_{y}^{-1}(\omega) \mathbf{S}_{z}(\omega)\right)+\operatorname{tr}\left[\mathbf{S}_{z}^{-1}(\omega)\left(\mathbf{S}_{y}(\omega)-\mathbf{S}_{z}(\omega)\right)\right]\right) d \omega .
$$

Using the relative entropy rate as a tool to quantitatively define the concept of model proximity, our simulation strategy can be formalized as follows:

Let $\{y(k), k \in \mathbb{Z}\}$ be the Gaussian random process taking values in $\mathbb{R}^{2 L}$ that we want to generate, find the positive definite matrix $\mathbf{S}_{y}^{o}(\omega)$ that solves

$$
\begin{cases}\underset{\mathbf{S}_{y}(\omega)}{\operatorname{minimize}} & D_{r}(y \| \tilde{h}) \\
\text { subject to } & \mathbb{F}_{n}\left(\mathbf{S}_{y}(\omega)\right)=\left\{\begin{array}{l}
\alpha_{n} \text { (scalar constraint) } \\
\boldsymbol{\Sigma}_{n} \text { (matrix constraint) }
\end{array}\right. \\
& n \in\left\{0, \cdots, N_{c}-1\right\},\end{cases}
$$

where $N_{c}$ denotes the number of constraints on the channel second-order statistics, $\mathbb{F}_{n}$ is some userspecified functional which is either equal to a scalar $\alpha_{n}$ or to a $2 L \times 2 L$ matrix $\boldsymbol{\Sigma}_{n}$, also specified by the user. The overall simulation methodology is illustrated in Fig. 2-(b). Note that constraints only apply to the zero-mean random components, the channel trend is kept identical to that of the sea-probed channel.

\section{B. Constraint on the channel Doppler spread}

To illustrate our modeling approach, we first consider constraints on the channel Doppler spread. The average Doppler spread $\sigma_{d}$ is a measure (in $\mathrm{Hz}$ ) of the spectral broadening caused by the time-varying nature of the channel and is here defined as

$$
\sigma_{d}=\frac{F_{s}^{(t)}}{2 \pi} \sqrt{\frac{\int_{-\pi}^{\pi} \omega^{2} \operatorname{tr}\left[\mathbf{S}_{\tilde{h}}(\omega)\right] d \omega}{\int_{-\pi}^{\pi} \operatorname{tr}\left[\mathbf{S}_{\tilde{h}}(\omega)\right] d \omega}}
$$

where $F_{s}^{(t)}$ is the sampling frequency of the TVIR along the time axis. Note that common definitions of the Doppler spread [33, Eq. 3.13b] are defined with respect to the center of gravity of the Doppler spectrum, i.e., $\int_{-\pi}^{\pi} \omega \operatorname{tr}\left[\mathbf{S}_{\tilde{h}}(\omega)\right] d \omega$. This term does not appear in (13) because, as a consequence of property (7), it is always null.

A particular value of Doppler spread can be specified by controlling the channel energy as well as the second moment of the cross-spectral density matrix. More precisely, the new model $\mathbf{S}_{y}(\omega)$ can be 
constrained to satisfy

$$
\begin{gathered}
\int_{-\pi}^{\pi} \operatorname{tr}\left[\mathbf{S}_{y}(\omega)\right] d \omega=\alpha_{0}, \\
\int_{-\pi}^{\pi} \omega^{2} \operatorname{tr}\left[\mathbf{S}_{y}(\omega)\right] d \omega=\alpha_{1} .
\end{gathered}
$$

In practice, the channel energy can be normalized so that the Doppler spread is fully expressed by $\alpha_{1}$.

Problem (12) with constraints (14) and (15) can be solved using the method of Lagrange multipliers. According to (11) and since $\frac{-1}{4 \pi} \int_{-\pi}^{\pi} \operatorname{tr}\left[\mathbf{S}_{\tilde{h}}^{-1}(\omega) \mathbf{S}_{\tilde{h}}(\omega)\right] d \omega$ plays no role in the optimization, the Lagrangian is expressed as

$$
\begin{aligned}
& L\left(\mathbf{S}_{y}, \lambda_{0}, \lambda_{1}\right)= \\
& \int\left(\log \frac{\operatorname{det} \mathbf{S}_{\tilde{h}}(\omega)}{\operatorname{det} \mathbf{S}_{y}(\omega)}+\operatorname{tr}\left[\mathbf{S}_{\tilde{h}}^{-1}(\omega) \mathbf{S}_{y}(\omega)\right]\right) d \omega+ \\
& \lambda_{0}\left(\int \operatorname{tr}\left[\mathbf{S}_{y}(\omega)\right] d \omega-\alpha_{0}\right)+\lambda_{1}\left(\int \omega^{2} \operatorname{tr}\left[\mathbf{S}_{y}(\omega)\right] d \omega-\alpha_{1}\right),
\end{aligned}
$$

where $\lambda_{0}$ and $\lambda_{1}$ are the Lagrange multipliers.

Using the fact that, for the positive definite matrix $\mathbf{S}_{y}(\omega)$, the directional derivative of $\log \operatorname{det}\left(\mathbf{S}_{y}(\omega)\right)$ in direction $\delta \mathbf{S}_{y}$ is equal to $\operatorname{tr}\left[\mathbf{S}_{y}^{-1}(\omega) \delta \mathbf{S}_{y}(\omega)\right]$ [34, Sec. 4, Th. 2], it can be shown that the directional derivative of the Lagrangian in direction $\delta \mathbf{S}_{y}$ satisfies

$$
\delta L\left(\mathbf{S}_{y}, \lambda_{0}, \lambda_{1} ; \delta \mathbf{S}_{y}\right)=\int \operatorname{tr}\left[\left(\mathbf{S}_{\tilde{h}}^{-1}(\omega)-\mathbf{S}_{y}^{-1}(\omega)+\left(\lambda_{0}+\lambda_{1} \omega^{2}\right) \mathbf{I}_{2 L}\right) \delta \mathbf{S}_{y}(\omega)\right] d \omega,
$$

where $\mathbf{I}_{2 L}$ denotes a $2 L \times 2 L$ identity matrix. Setting this derivative to zero leads to the following solution ${ }^{4}$

$$
\mathbf{S}_{y}^{o}(\omega)=\left[\mathbf{S}_{\tilde{h}}^{-1}(\omega)+\left(\lambda_{0}+\lambda_{1} \omega^{2}\right) \mathbf{I}_{2 L}\right]^{-1}
$$

The multipliers $\lambda_{0}$ and $\lambda_{1}$ are then obtained by solving the equations (14) and (15). A numerical solution can be found using a Newton algorithm as presented in Appendix B.

\section{Constraint on the level of covariance between channel taps}

To simplify the statistical characterization of linear time-varying channels, the uncorrelated scattering assumption is very often invoked. In our context, this assumption would imply that the four submatrices of (5) are diagonals (i.e., absence of correlation between two random components $\tilde{h}_{l}(k)$ and $\tilde{h}_{p}(k)$, with $l \neq p$ ). In practice, this property is not satisfied for scatterers with close coordinates in the delay-Doppler

\footnotetext{
${ }^{4}$ Thanks to the Woodbury identity, the computation of the inverse matrix $\mathbf{S}_{\tilde{h}}^{-1}(\omega)$ can be avoided and (18) can also be written as $\mathbf{S}_{y}^{o}(\omega)=\mathbf{S}_{\tilde{h}}(\omega)\left(\mathbf{I}_{2 L}-\left[\mathbf{I}_{2 L}+\left(\lambda_{0}+\lambda_{1} \omega^{2}\right) \mathbf{S}_{\tilde{h}}(\omega)\right]^{-1}\left(\lambda_{0}+\lambda_{1} \omega^{2}\right) \mathbf{S}_{\tilde{h}}(\omega)\right)$.
} 
domain because they usually result from reflections on the same physical body and are thus correlated [35]. In addition, from the receiver perspective, the transmission channel also includes the acoustic frontend as well as filters that induce extra correlation between channel taps. Examples of UAC channels with correlated scattering are discussed in [36], [37].

Using the definition of the cross-spectral density matrix $\mathbf{S}_{\tilde{h}}(\omega)$, the covariance coefficient between two channel elements can be expressed as

$$
\rho_{i j} \triangleq \frac{\int_{-\pi}^{\pi}\left[\mathbf{S}_{\tilde{h}}(\omega)\right]_{i j} d \omega}{\sqrt{\int_{-\pi}^{\pi}\left[\mathbf{S}_{\tilde{h}}(\omega)\right]_{i i} d \omega \int_{-\pi}^{\pi}\left[\mathbf{S}_{\tilde{h}}(\omega)\right]_{j j} d \omega}} .
$$

As an example, Fig. 3 shows the covariance coefficients for the channel of Fig. 1.

From (19), it can be seen that the level of covariance between taps (and also between the real and the imaginary part of a single tap) can be controlled by forcing the cross-spectral density matrix of the simulated channel to satisfy

$$
\int_{-\pi}^{\pi} \mathbf{S}_{y}(\omega) d \omega=\boldsymbol{\Sigma}
$$

where $\boldsymbol{\Sigma}$ is the user-defined covariance matrix of the output process. By analogy with (6), $\boldsymbol{\Sigma}$ can be seen as a block matrix with, for instance, $\left[\boldsymbol{\Sigma}^{\Re, \Im}\right]_{l p}=\int_{-\pi}^{\pi}\left[\mathbf{S}_{y}^{\Re, \Im}(\omega)\right]_{l p} d \omega=\mathbb{E}\left\{\Re\left\{y_{l}(k)\right\} \Im\left\{y_{p}(k)\right\}\right\}$.

Problem (12) with constraint (20) can also be solved using the method of Lagrange multipliers. Recalling that the inner product in the vector space of symmetric matrices is defined as $\langle\mathbf{A}, \mathbf{B}\rangle=\operatorname{tr}[\mathbf{A B}]$, the Lagrangian is expressed as

$$
L\left(\mathbf{S}_{y}, \boldsymbol{\Lambda}\right)=\int\left(\log \frac{\operatorname{det} \mathbf{S}_{\tilde{h}}(\omega)}{\operatorname{det} \mathbf{S}_{y}(\omega)}+\operatorname{tr}\left[\mathbf{S}_{\tilde{h}}^{-1}(\omega) \mathbf{S}_{y}(\omega)\right]+\operatorname{tr}\left[\boldsymbol{\Lambda} \mathbf{S}_{y}(\omega)\right]\right) d \omega-\operatorname{tr}[\boldsymbol{\Lambda} \boldsymbol{\Sigma}]
$$

where $\Lambda$ is a $2 L \times 2 L$ symmetric matrix that denotes the Lagrange multipliers. Following the same approach as in Section III-B, the optimal cross-spectral density matrix is found to be

$$
\mathbf{S}_{y}^{o}(\omega)=\left[\mathbf{S}_{\tilde{h}}^{-1}(\omega)+\boldsymbol{\Lambda}\right]^{-1}
$$

A numerical solution to find $\boldsymbol{\Lambda}$ is presented in Appendix C.

The way constraint (20) is applied allows the user to have a full control on the inter and intra channel tap covariation. However, it is so detailed that it may sometimes be difficult to set numerical values for every elements of $\boldsymbol{\Sigma}$. An alternative way of specifying covariance values is to reformulate the covariance between taps in terms of magnitude and phase correlations. For instance, consider a particular case of 

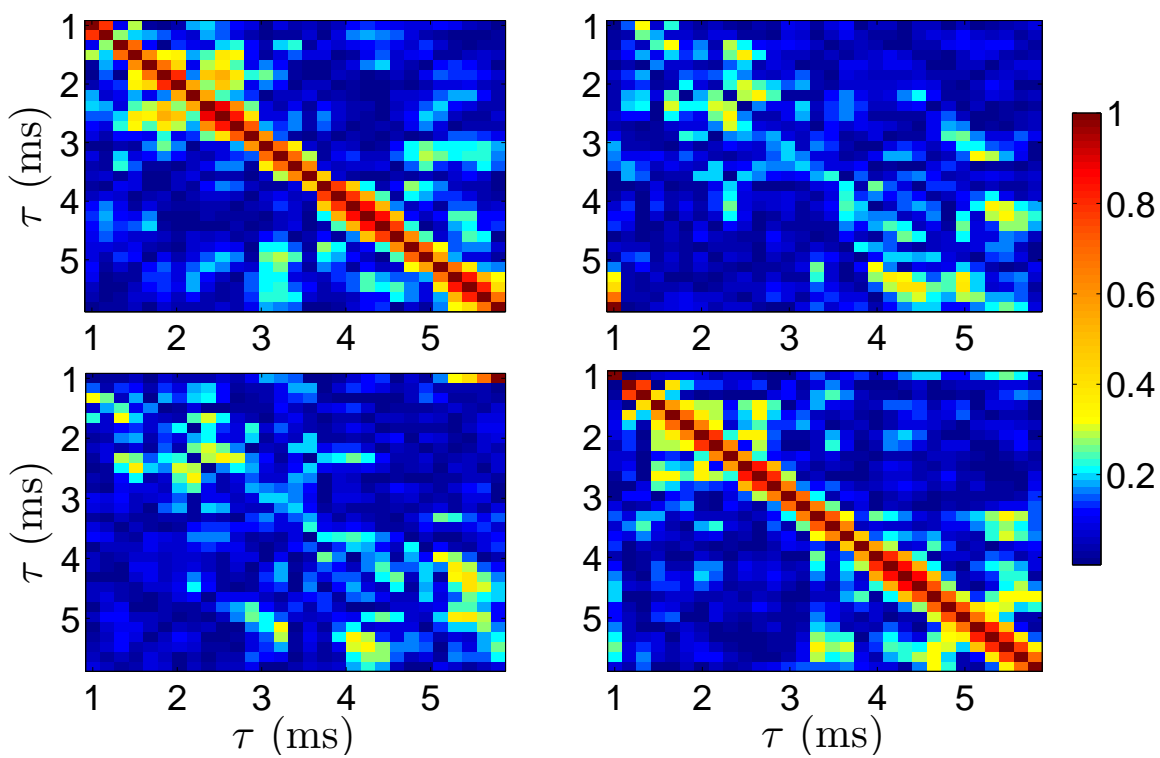

Fig. 3. Covariance coefficients for the channel of Fig. 1. The covariance matrix is partitioned into four submatrices similarly to definition (5).

jointly $\operatorname{proper}^{5}$ random taps with for all $l$ and $p$

$$
\begin{aligned}
& \mathbb{E}\left\{\Re\left\{y_{l}(k)\right\} \Im\left\{y_{p}(k)\right\}\right\}=0 \\
& \mathbb{E}\left\{\Re\left\{y_{l}(k)\right\} \Re\left\{y_{p}(k)\right\}\right\}=\mathbb{E}\left\{\Im\left\{y_{l}(k)\right\} \Im\left\{y_{p}(k)\right\}\right\} .
\end{aligned}
$$

Define each tap $l$ in polar form as $y_{l}(k)=a_{l}(k) e^{i \theta_{l}(k)}$. In this case, the covariance between taps can be fully specified by $\mathbb{E}\left\{a_{l}(k) a_{p}(k)\right\}$ and $\mathbb{E}\left\{\cos \left(\theta_{l}(k)\right) \cos \left(\theta_{p}(k)\right)\right\}$ since, from (23) and (24), $\boldsymbol{\Sigma}$ satisfies ${ }^{6}$

$$
\begin{aligned}
\boldsymbol{\Sigma}^{\Re, \Im} & =\boldsymbol{\Sigma}^{\Im, \Re}=\mathbf{0}, \\
{\left[\boldsymbol{\Sigma}^{\Re, \Re}\right]_{l p} } & =\left[\boldsymbol{\Sigma}^{\Im, \Im}\right]_{l p}=\mathbb{E}\left\{a_{l}(k) a_{p}(k)\right\} \mathbb{E}\left\{\cos \left(\theta_{l}(k)\right) \cos \left(\theta_{p}(k)\right)\right\} .
\end{aligned}
$$

Such a reformulation can be relevant to examine the effect of amplitude or phase correlation alone. In particular, phase-correlated channels represent an important class of acoustic channels and controlling phase correlation may be useful to characterize the behavior of phase-locked loops commonly used in receivers.

${ }^{5}$ Refer to [38, Sec. III-B] for a definition of proper random processes.

${ }^{6}$ Note that $(24)$ implies $\mathbb{E}\left\{\cos \left(\theta_{l}(k)\right) \cos \left(\theta_{p}(k)\right)\right\}=\mathbb{E}\left\{\sin \left(\theta_{l}(k)\right) \sin \left(\theta_{p}(k)\right)\right\}$. 


\section{ILLUSTRATIONS}

The parametric replay-based simulation strategy is here illustrated through bit error rate (BER) measurements of a QPSK communication scheme put through a shallow water channel whose sea-probed TVIR is shown in Fig. 1. This TVIR has been extracted from an experiment conducted in 2007 in the Bay of Brest. The water depth varied between approximately 10 to $20 \mathrm{~m}$ over a $1 \mathrm{~km}$ track and both the transmitter and the receiver were immersed $5 \mathrm{~m}$ below the sea surface. This channel was probed at a center frequency of $17.5 \mathrm{kHz}$ with a $2.9 \mathrm{kBd}$ QPSK signal (for more details, refer to [4, Sec. II]). The average Doppler spread of the in-situ TVIR is approximately $0.7 \mathrm{~Hz}$ and the Rice factor $\kappa$, defined as the power ratio between the deterministic trend and the random component, i.e. :

$$
\kappa=\frac{1}{K} \frac{\sum_{k=0}^{K-1} \sum_{l=0}^{L-1}\left|\bar{h}_{l}(k)\right|^{2}}{\sum_{l=0}^{L-1} \mathbb{E}\left\{\left|\tilde{h}_{l}(k)\right|^{2}\right\}},
$$

is equal to $6.5 \mathrm{~dB}$ approximately.

The receiver implemented for the simulations is an adaptive decision-feedback equalizer (DFE) with joint phase tracking [39] and its performance is assessed for various channel Doppler spreads and several levels of covariance between channel taps. The DFE operates with $2 L$ feedforward taps and $L$ feedback taps. For transmit data of power $P_{d}$, filter coefficients are updated with LMS algorithms of step-sizes $\mu_{\mathrm{ff}}=1 /\left(10 P_{d} \times(2 L+1)\right)$ and $\mu_{\mathrm{fb}}=1 /\left(10 P_{d} \times(L+1)\right)$ for the feedforward filter and the feedback filter, respectively. The first 1000 symbols fed into the DFE are training symbols used for convergence of the adaptive filters. For the remaining symbols used for BER computation, the DFE is decision-directed. No attempt is made to optimize the receiver's parameters or to compare it with other receiver structures. The objective is not to evaluate communication performance, but to illustrate how our simulator can help modem designers. Each BER value is obtained from 100 realizations of $30 \mathrm{sec}$. long TVIRs.

\section{A. Influence of the channel Doppler spread}

Fig. 4 represents the auto-spectral density functions, a.k.a. Doppler spectra, of the two most powerful random taps of the TVIR shown in Fig. 1 (i.e., real and imaginary parts of the taps at $\tau \approx 2 \mathrm{~ms}$ and $\tau \approx 3.8 \mathrm{~ms}$ in Fig. 1). The channel energy, as defined in (14), is set to $\alpha_{0}=2 \pi$. Solid lines corresponds to the original spectra estimated from the in-situ TVIR. Other spectra are represented for two different constraints $\alpha_{1}$. Changing the average Doppler spread through the value $\alpha_{1}$ affects all taps, but in different ways. Dashed lines indicate the output of the optimization procedure (12) when the taps fluctuations are artificially slowed down. In this case, the average Doppler spread (13) is halved. As for the dash-dotted lines, they correspond to faster taps fluctuations with an average Doppler spread multiplied by 1.5. It can 


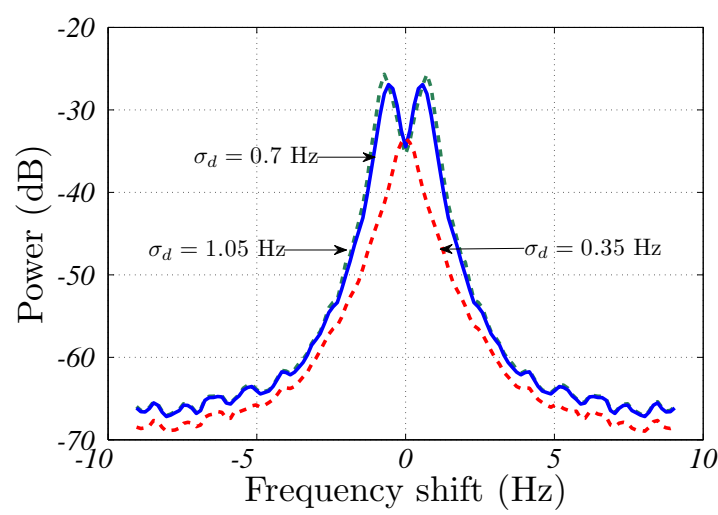

(a)

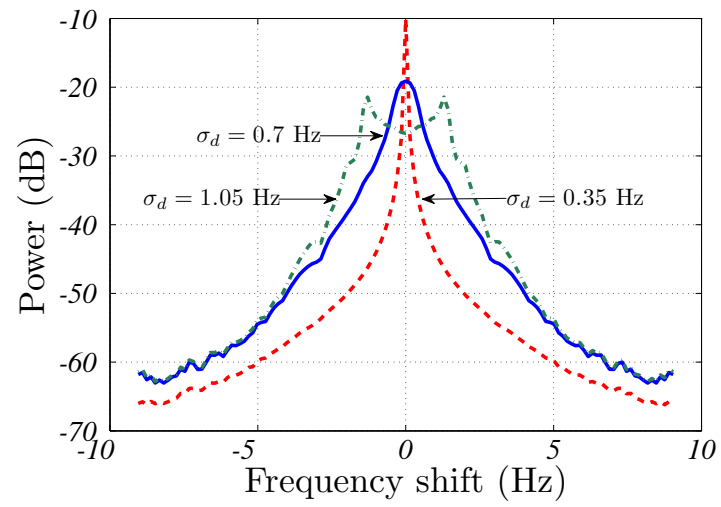

(c)

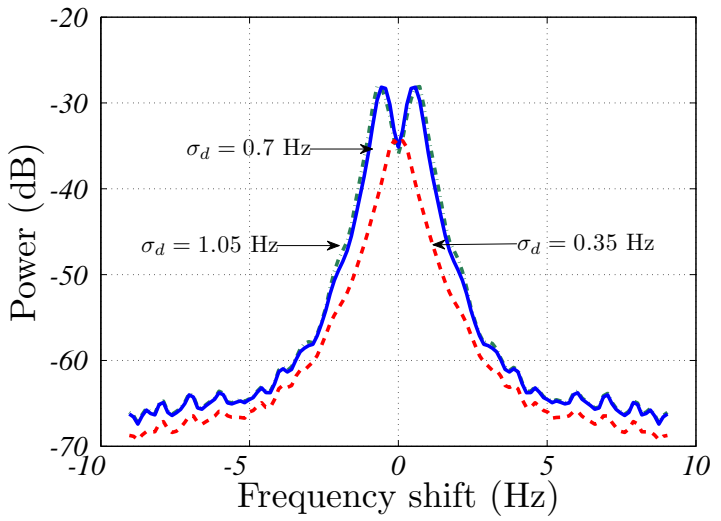

(b)

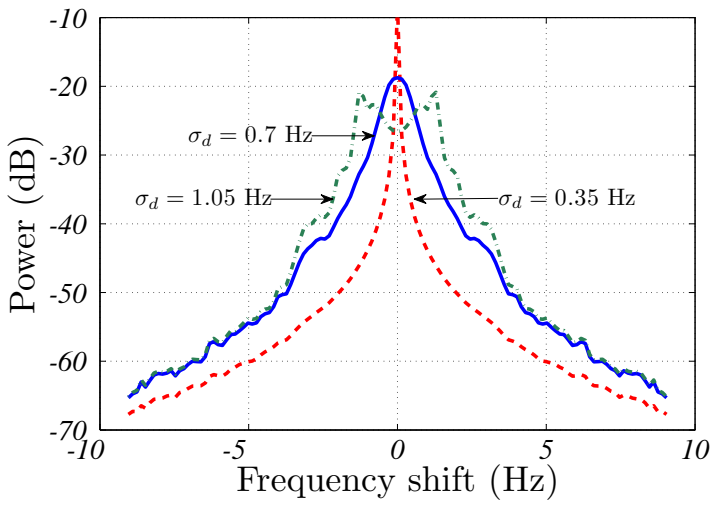

(d)

Fig. 4. Doppler spectra of taps at $\tau \approx 2 \mathrm{~ms}$ and $\tau \approx 3.8 \mathrm{~ms}$ in Fig. 1 for different constraints on the average Doppler spread. (a) real part, (b) imaginary part of taps at $\tau \approx 2 \mathrm{~ms}$. (c) real part, (d) imaginary part of taps at $\tau \approx 3.8 \mathrm{~ms}$. Solid lines correspond to the original spectra estimated from the in-situ TVIR ( $\sigma_{d}=0.7 \mathrm{~Hz}$ ). Dashed lines indicate the output of the optimization procedure for $\sigma_{d}=0.35 \mathrm{~Hz}$ and dash-dotted lines for $\sigma_{d}=1.05 \mathrm{~Hz}$.

be observed that the Doppler spread modification changes the shape of the Doppler spectra. For instance, the Doppler spectra of Fig. 4 (c) and (d) get very peaky for small average Doppler spread and become multimodal as this spread increases. It is also interesting to note that the tap at $\tau \approx 3.8 \mathrm{~ms}$ is more affected by the Doppler spread changes than the tap at $\tau \approx 2 \mathrm{~ms}$. This is explained by the fact that the random part of the tap at $\tau \approx 3.8 \mathrm{~ms}$ is $8 \mathrm{~dB}$ more powerful than its counterpart at at $\tau \approx 2 \mathrm{~ms}$. Since the constraint is set on the average Doppler spread, it is less demanding in term of entropy change to act on the most powerful taps.

Fig. 5 shows the BER of an uncoded $1.95 \mathrm{kBd}$ QPSK system as a function of Eb/N0 for several constraints on the Doppler spread. For simplicity, the noise is modeled as an additive white Gaussian 


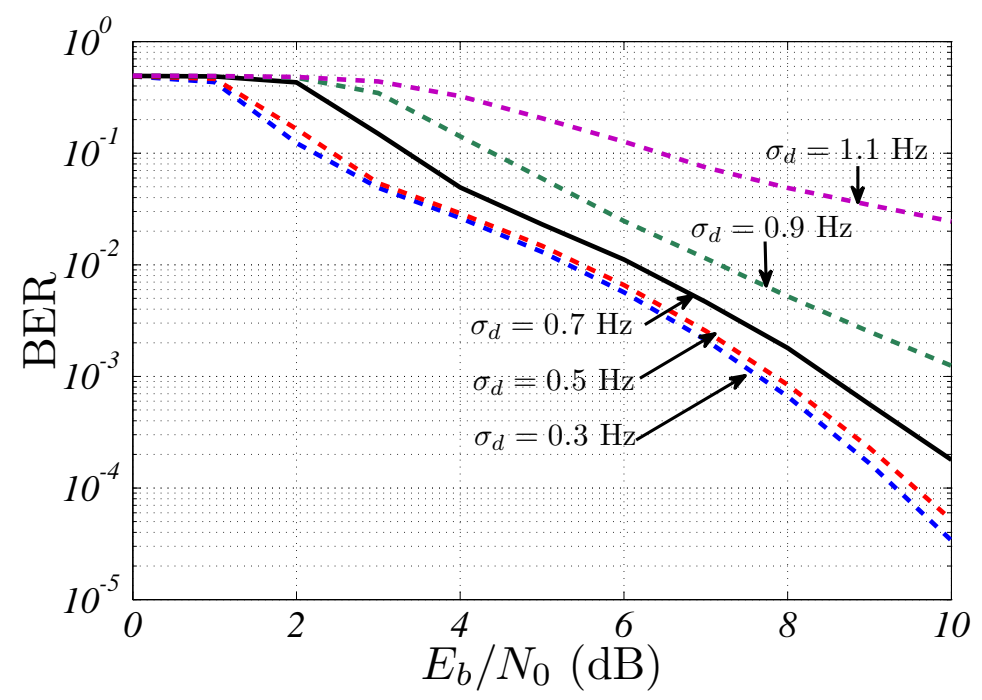

Fig. 5. BER as a function of Eb/N0 for several constraints on the average Doppler spread.

noise. Not surprisingly, the Doppler spread strongly impacts the receiver's performance. As this spread increases, the adaptive DFE gets some difficulties to track the channel fluctuations. Such figures of merit can be useful when designing a receiver, either to bound the range of Doppler spread for which the receiver's performance is acceptable, or to tune some parameters (especially the LMS step-sizes jointly with the filters order in our example) to make the receiver adapted to some specific environment.

\section{B. Influence of the channel taps covariance}

To illustrate the impact of the level of covariance between taps, we constrain the output process $\{y(k)\}$ to have a covariance matrix $\boldsymbol{\Sigma}$, as defined in (20), controlled by a simple scalar. The methodology of Sec. III-C is general and applies to any structure of covariance matrix but, to ease the illustration, a canonical covariance model is chosen. More precisely, for the simulation, matrix $\boldsymbol{\Sigma}$ takes the following form

$$
[\boldsymbol{\Sigma}]_{l l}=\int_{-\pi}^{\pi}\left[\mathbf{S}_{\tilde{h}}(\omega)\right]_{l l} d \omega
$$

and, for $l \neq p$,

$$
\frac{[\boldsymbol{\Sigma}]_{l p}}{\sqrt{[\boldsymbol{\Sigma}]_{l l}[\boldsymbol{\Sigma}]_{p p}}}=\rho_{0},
$$

where $\rho_{0}$ is some constant between 0 and 1. (28) indicates that the power of the simulated taps is the same as the original taps and (29) means that each pair of simulated taps has the same level of covariance $\rho_{0}$. 


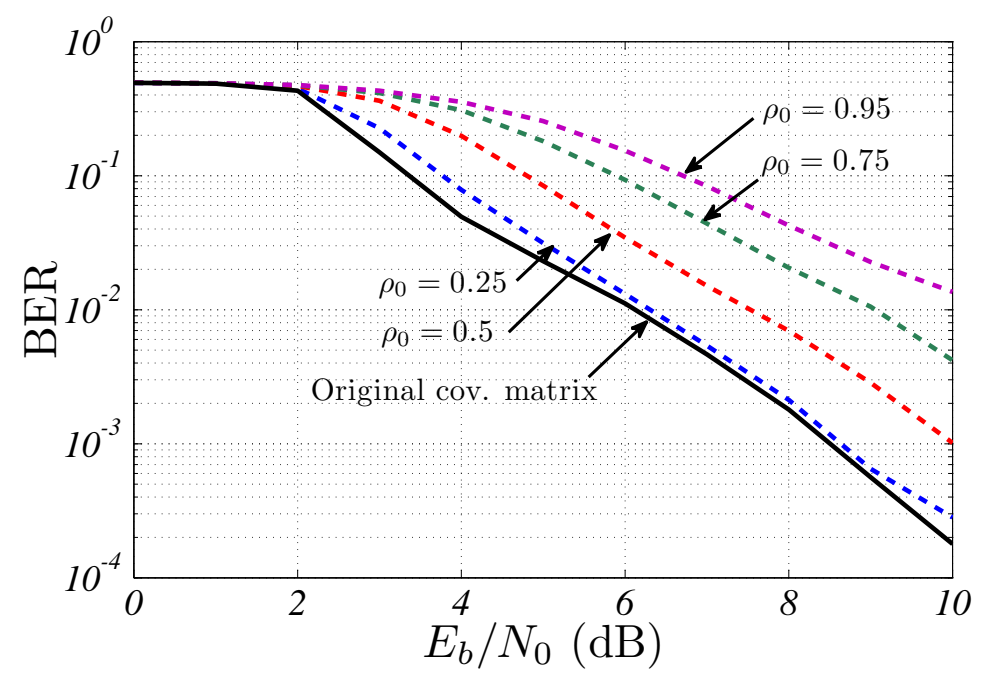

Fig. 6. BER as a function of Eb/N0 for several constraints on the covariance $\rho_{0}$.

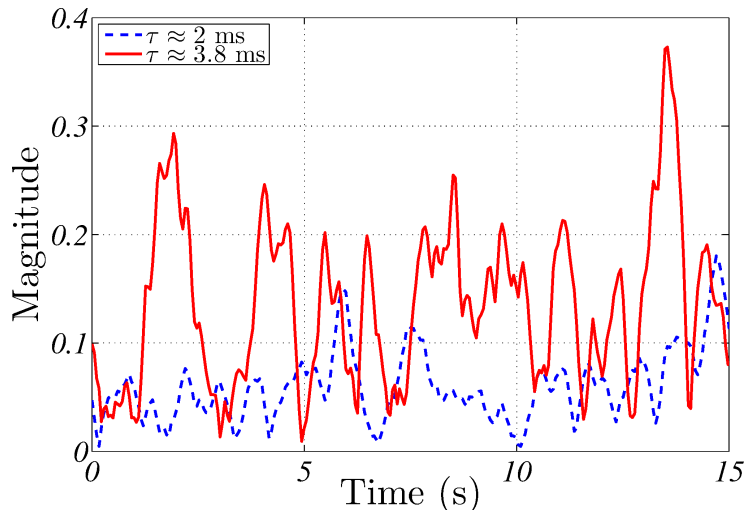

(a)

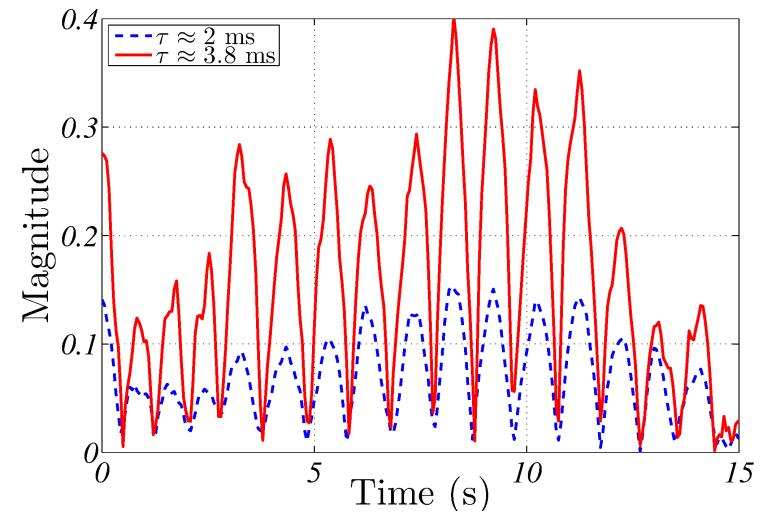

(b)

Fig. 7. Examples of sample paths (in linear scale) of the channel random components $\left|y_{l}(k)\right|$ as generated by the simulator with constraints on the covariance $\rho_{0}$. (a) $\rho_{0}=0.25$, (b) $\rho_{0}=0.95$. For both figures, only taps at $\tau \approx 2 \mathrm{~ms}$ and $\tau \approx 3.8 \mathrm{~ms}$ are shown.

Fig. 6 shows the effects of the covariance coefficient $\rho_{0}$ on the BER. ${ }^{7}$ As $\rho_{0}$ increases, the channel taps tend to fade simultaneously more frequently which may create recurrent drop of the instantaneous signal-to-noise ratio (SNR) at the receiver. The effect of correlation is clearly visible in Fig. 7 where outputs $\left|y_{l}(k)\right|$ of the simulator are shown for two levels of correlation, $\rho_{0}=0.25$ and $\rho_{0}=0.95$. When

${ }^{7}$ Note that a covariance coefficient $\rho_{0}$ as large as 0.95 is quite extreme and is quite unlikely to be encountered at sea. It is shown here only to illustrate the capabilities of the proposed simulation methodology. 


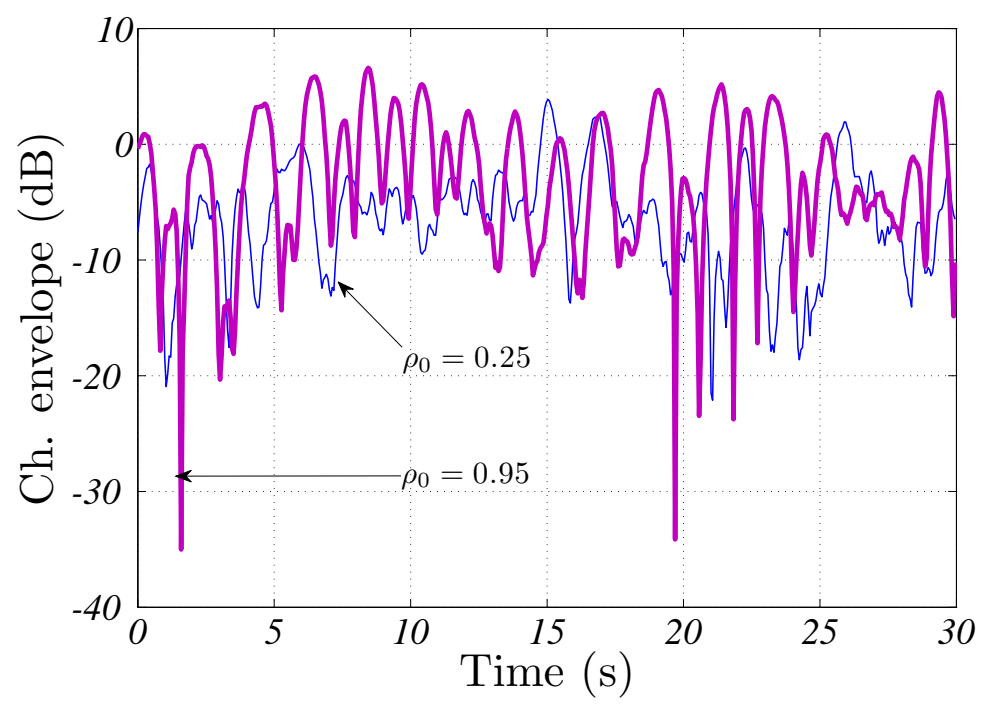

Fig. 8. Comparison of the channel envelope fluctuations for $\rho_{0}=0.25$ and $\rho_{0}=0.95$. This envelope includes the trend and is defined as $r(k)=\left|\sum_{l} \bar{h}_{l}\left(k-\left\lfloor\frac{l F_{s}^{(t)}}{F_{s}^{(\tau)}}\right\rfloor\right)+y_{l}\left(k-\left\lfloor\frac{l F_{s}^{(t)}}{F_{s}^{(\tau)}}\right\rfloor\right)\right|$ where $F_{s}^{(t)}$ and $F_{s}^{(\tau)}$ are the sampling frequencies of the TVIR along the time and delay axis, respectively. Deep fades are observed quite frequently for $\rho_{0}=0.95$, which may result in recurrent temporary failure of communication.

the covariance is large, the channel random components tend to oscillate coherently. This coherence leads to deep fades that can be quite frequent for large values of $\rho_{0}$ (see Fig. 8). This phenomenon creates burst errors due to a lack of multipath diversity and consequently increases the BER. Simulations with controlled covariance matrices can therefore be of interest to assess the robustness of coding mechanisms designed to combat such a type of errors.

\section{Conclusions And Perspectives}

UAC channel simulation as defined in the proposed parametric replay-based framework is a way to find a good compromise between simulation realism, parameterization simplicity and flexibility. Thanks to the formalism of relative entropy minimization between random processes, statistical properties of the simulated channel can be controlled, while being close to realistic TVIRs. Illustrations with userdefined values of Doppler spread or taps covariance have shown that such a simulation approach can help modem designers either to tune receivers or to better define their operating range. The main limitation of the proposed simulation strategy holds in the absence of control of the transmission geometry, i.e, the number of taps as well as their respective delay remain unchanged. Moreover, while Doppler spread is a well-studied phenomenon, experimental results on the actual covariance between taps are very scarce in 
the literature. This can make it difficult to specify a realistic covariance matrix $\boldsymbol{\Sigma}$. Further investigations have to be conducted to better identify the kinds of covariance structure that can be expected at sea.

Extension of this work to SIMO or MIMO channels is possible by adding the space dimension in the definition of the cross-spectral density matrix (5). In this scenario, the theoretical framework would remain the same and spatial correlations could be controlled. However, limitations might appear in the numerical resolution of (22) due to the dimension increase. Ultimately, it might also be relevant to merge physics-based model with replay-based simulation in order to link environmental conditions to statistical constraints such as (15) and (20).

\section{ACKNOWLEDGMENTS}

The authors are grateful to the DGA-TN for providing the experimental data used in this paper.

\section{APPENDIX A}

\section{OVERVIEW OF MULTIVARIATE GAUSSIAN PROCESSES SIMULATION}

Let $\left\{x(t) \triangleq\left[x_{1}(t), x_{2}(t), \cdots, x_{M}(t)\right]\right\}$ be a real-valued Gaussian process taking values in $\mathbb{R}^{M}$ with cross-spectral density matrix $\mathbf{S}_{x}(\omega)$. Because $\mathbf{S}_{x}(\omega)$ is hermitian, using Cholesky's decomposition, it can be factorized as

$$
\mathbf{S}_{x}(\omega)=\mathbf{H}(\omega) \mathbf{H}^{\dagger}(\omega)
$$

where $\mathbf{H}(\omega)$ is a lower triangular matrix. If the elements of $\mathbf{H}(\omega)$ are written in polar form as

$$
[\mathbf{H}(\omega)]_{l p}=\left|[\mathbf{H}(\omega)]_{l p}\right| e^{j \theta_{l p}(\omega)},
$$

then each stochastic process $x_{m}(t), m=1,2, \cdots, M$ can be simulated for large $N$ as ${ }^{8}$ [22]

$$
x_{m}(t) \approx \sqrt{\frac{2 \Delta \omega}{\pi}} \sum_{l=1}^{m} \sum_{k=1}^{N}\left|\left[\mathbf{H}\left(\omega_{l k}\right)\right]_{m l}\right| \cos \left(\omega_{l k} t-\theta_{m l}\left(\omega_{l k}\right)+\phi_{l k}\right),
$$

where

$$
\omega_{k l}=\left(l-1+\frac{k}{M}\right) \Delta \omega, l=1,2, \cdots, M ; k=1,2, \cdots, N
$$

and $\Delta \omega=\omega_{u} / N$, with $\omega_{u}$ is the upper cutoff frequency beyond which the entries of $\mathbf{S}_{x}(\omega)$ are assumed to be zero. The phase terms $\phi_{l k}$ are independent and uniformly distributed over the interval $[0,2 \pi]$. The computational cost of (32) can be reduced by making use of the FFT technique. Note that the method implemented in Section IV is the one detailed in [24], which is an improved version of the original method presented in [22].

\footnotetext{
${ }^{8}$ For simplicity, $x_{m}(t)$ also denotes the sample function.
} 


\section{APPENDIX B}

\section{NUMERICAL SOLUTION OF (18)}

The proposed solution is based on a backtracking line search algorithm and is inspired from the Newton-like algorithm presented in [30], [31].

To find the Lagrange multipliers $\Lambda \triangleq\left(\lambda_{0}, \lambda_{1}\right)$, we first resort to the duality principle. Consider

$$
L\left(\mathbf{S}_{y}^{o}, \Lambda\right)=\int \log \operatorname{det}\left(\mathbf{S}_{\tilde{h}}^{-1}(\omega)+\gamma(\Lambda, \omega) \mathbf{I}_{2 L}\right)+\log \operatorname{det}\left(\mathbf{S}_{\tilde{h}}(\omega)\right) d \omega+2 L-\alpha_{0} \lambda_{0}-\alpha_{1} \lambda_{1},
$$

where $\gamma(\Lambda, \omega) \triangleq \lambda_{0}+\lambda_{1} \omega^{2}$. Note that (34) is obtained by injecting (18) in (16). According to the duality theory ${ }^{9}$, our primal problem can be restated as the following dual problem:

$$
\text { Find } \Lambda \in \mathcal{L}_{+} \text {minimizing } J(\Lambda) \triangleq-L\left(\mathbf{S}_{y}^{o}, \Lambda\right) \text {, }
$$

where $\mathcal{L}_{+} \triangleq\left\{\left(\lambda_{0}, \lambda_{1}\right) \mid \mathbf{S}_{y}^{o}>0\right\}$. Note that $\mathbf{S}_{y}^{o}$ has to be positive definite since it is a cross-spectral density matrix (see also (7)).

Problem (35) is then solved by applying the following iterative procedure:

1) Set the initial condition $\Lambda_{0}=(0,0)$

2) At iteration $i$, compute the Newton search direction

$$
d_{\Lambda_{i}}=-\left[\mathcal{H} J_{\Lambda_{i}}\right]^{-1} \nabla J_{\Lambda_{i}}
$$

where $\mathcal{H} J_{\Lambda_{i}}$ and $\nabla J_{\Lambda_{i}}$ are the Hessian and the gradient of $J$ at $\Lambda_{i}$, respectively. $\mathcal{H} J$. and $\nabla J$. can be computed using the following relations:

$$
\begin{gathered}
\frac{\partial J}{\partial \lambda_{0}}=\alpha_{0}-\int \operatorname{tr}\left[\left(\mathbf{S}_{\tilde{h}}^{-1}(\omega)+\gamma(\Lambda, \omega) \mathbf{I}_{2 L}\right)^{-1}\right] d \omega \\
\frac{\partial J}{\partial \lambda_{1}}=\alpha_{1}-\int \omega^{2} \operatorname{tr}\left[\left(\mathbf{S}_{\tilde{h}}^{-1}(\omega)+\gamma(\Lambda, \omega) \mathbf{I}_{2 L}\right)^{-1}\right] d \omega \\
\frac{\partial^{2} J}{\partial \lambda_{0} \partial \lambda_{0}}=\int \operatorname{tr}\left[\left(\mathbf{S}_{\tilde{h}}^{-1}(\omega)+\gamma(\Lambda, \omega) \mathbf{I}_{2 L}\right)^{-2}\right] d \omega \\
\frac{\partial^{2} J}{\partial \lambda_{1} \partial \lambda_{1}}=\int \omega^{4} \operatorname{tr}\left[\left(\mathbf{S}_{\tilde{h}}^{-1}(\omega)+\gamma(\Lambda, \omega) \mathbf{I}_{2 L}\right)^{-2}\right] d \omega \\
\frac{\partial^{2} J}{\partial \lambda_{0} \partial \lambda_{1}}=\int \omega^{2} \operatorname{tr}\left[\left(\mathbf{S}_{\tilde{h}}^{-1}(\omega)+\gamma(\Lambda, \omega) \mathbf{I}_{2 L}\right)^{-2}\right] d \omega \\
\frac{\partial^{2} J}{\partial \lambda_{1} \partial \lambda_{0}}=\frac{\partial^{2} J}{\partial \lambda_{0} \partial \lambda_{1}}
\end{gathered}
$$

\footnotetext{
${ }^{9}$ The existence of the dual problem as well as the convergence of the proposed algorithm is out of the scope of this paper. For details on these aspects, the reader is referred to [30], [31].
} 
3) Set $t_{i}^{0}=1$, and let $t_{i}^{k+1}=t_{i}^{k} / 2$ until both of the following conditions hold:

$$
\begin{aligned}
& \left(\mathbf{S}_{\tilde{h}}^{-1}(\omega)+\gamma\left(\Lambda_{i}+t_{i}^{k} d_{\Lambda_{i}}, \omega\right) \mathbf{I}_{2 L}\right)^{-1}>0, \\
& J\left(\Lambda_{i}+t_{i}^{k} d_{\Lambda_{i}}\right)<J\left(\Lambda_{i}\right)+\beta t_{i}^{k} d_{\Lambda_{i}}^{T} \nabla J\left(\Lambda_{i}\right),
\end{aligned}
$$

where $\beta \in(0,1 / 2)$. The first condition guarantees that $\Lambda_{i}+t_{i}^{k} d_{\Lambda_{i}}$ belongs to $\mathcal{L}_{+}$and the second one is the Armijo condition useful to determine the maximum amount to move along the search direction $d_{\Lambda_{i}}$ [40, Ch. 3].

4) Set $\Lambda_{i+1}=\Lambda_{i}+t_{i}^{k} d_{\Lambda_{i}}$.

5) Repeat steps 2, 3 and 4 until $\left\|\nabla J\left(\Lambda_{i}\right)\right\|_{2}<\epsilon$, where $\epsilon$ is a small tolerance.

In all the simulations conducted in this paper, this numerical approach converged quickly. For all scenarios of Sec. IV-A, it took less than 10 iterations to get $\left(\lambda_{0}, \lambda_{1}\right)$ with $\epsilon$ set to $10^{-6}$. This represents a couple of seconds of processing time on a standard computer (CPU: $2.6 \mathrm{GHz}$, RAM: 4 Go) with a Matlab implementation.

\section{APPENDIX C}

\section{NUMERICAL SOLUTION OF (22)}

The approach used to find $\boldsymbol{\Lambda}$ in (22) is very similar to the one presented in Appendix B, except that the unknown is now a matrix, which changes the way the search direction is computed. Consider

$$
L\left(\mathbf{S}_{y}^{o}, \boldsymbol{\Lambda}\right)=\int \log \operatorname{det}\left(\mathbf{S}_{\tilde{h}}^{-1}(\omega)+\boldsymbol{\Lambda}\right)+\log \operatorname{det}\left(\mathbf{S}_{\tilde{h}}(\omega)\right) d \omega+2 L-\operatorname{tr}[\mathbf{\Lambda} \boldsymbol{\Sigma}],
$$

obtained by injecting (22) in (21). The primal problem is restated as the following dual problem:

$$
\text { Find } \boldsymbol{\Lambda} \in \mathcal{L}_{+} \text {minimizing } J(\boldsymbol{\Lambda}) \triangleq-L\left(\mathbf{S}_{y}^{o}, \mathbf{\Lambda}\right),
$$

where $\mathcal{L}_{+} \triangleq\left\{\boldsymbol{\Lambda} \mid \mathbf{S}_{y}^{o}>0\right\}$. The dual function $J(\boldsymbol{\Lambda})$ is then minimized by applying the following iterative procedure:

1) Set the initial condition $\boldsymbol{\Lambda}_{0}=0$.

2) At iteration $i$, compute the Newton search direction $\boldsymbol{\Delta}_{\boldsymbol{\Lambda}_{i}}$. This computation is not straightforward because the expression of the search direction in the form of (36) is not available. The unknown $\boldsymbol{\Delta}_{\boldsymbol{\Lambda}_{i}}$ is therefore obtained by solving the following equation

$$
\mathcal{H}_{\boldsymbol{\Delta}_{\Lambda_{i}}} J_{\Lambda_{i}}=-\nabla J_{\Lambda_{i}}
$$

where $\mathcal{H}_{\boldsymbol{\Delta}_{\boldsymbol{\Lambda}_{i}}} J_{\boldsymbol{\Lambda}_{i}}$ is the second variation of $J$ at $\boldsymbol{\Lambda}_{i}$ in direction $\boldsymbol{\Delta}_{\boldsymbol{\Lambda}_{i}}$. Using the fact that

$$
\delta J(\boldsymbol{\Lambda} ; \delta \boldsymbol{\Lambda})=\int \operatorname{tr}\left[\left(\boldsymbol{\Sigma}-\left(\mathbf{S}_{\tilde{h}}^{-1}(\omega)+\boldsymbol{\Lambda}\right)^{-1}\right) \delta \boldsymbol{\Lambda}\right] d \omega,
$$




$$
\delta J^{2}\left(\boldsymbol{\Lambda} ; \delta \boldsymbol{\Lambda}_{1}, \delta \boldsymbol{\Lambda}_{2}\right)=\int \operatorname{tr}\left[\left(\mathbf{S}_{\tilde{h}}^{-1}(\omega)+\boldsymbol{\Lambda}\right)^{-1} \delta \boldsymbol{\Lambda}_{2}\left(\mathbf{S}_{\tilde{h}}^{-1}(\omega)+\boldsymbol{\Lambda}\right)^{-1} \delta \boldsymbol{\Lambda}_{1}\right] d \omega
$$

(41) can explicitly be written as

$$
\int\left(\mathbf{S}_{\tilde{h}}^{-1}(\omega)+\boldsymbol{\Lambda}_{i}\right)^{-1} \boldsymbol{\Delta}_{\boldsymbol{\Lambda}_{i}}\left(\mathbf{S}_{\tilde{h}}^{-1}(\omega)+\boldsymbol{\Lambda}_{i}\right)^{-1} d \omega=\int\left(\mathbf{S}_{\tilde{h}}^{-1}(\omega)+\boldsymbol{\Lambda}_{i}\right)^{-1} d \omega-\boldsymbol{\Sigma} .
$$

3) Set $t_{i}^{0}=1$, and let $t_{i}^{k+1}=t_{i}^{k} / 2$ until both of the following conditions hold:

$$
\begin{array}{r}
{\left[\mathbf{S}_{\tilde{h}}^{-1}(\omega)+\boldsymbol{\Lambda}_{i}+t_{i}^{k} \boldsymbol{\Delta}_{\boldsymbol{\Lambda}_{i}}\right]^{-1}>0,} \\
J\left(\boldsymbol{\Lambda}_{i}+t_{i}^{k} \boldsymbol{\Delta}_{\boldsymbol{\Lambda}_{i}}\right)<J\left(\boldsymbol{\Lambda}_{i}\right)+\beta t_{i}^{k} \operatorname{tr}\left[\boldsymbol{\Delta}_{\boldsymbol{\Lambda}_{i}} \nabla J\left(\Lambda_{i}\right)\right],
\end{array}
$$

where $\beta \in(0,1 / 2)$.

4) Set $\boldsymbol{\Lambda}_{i+1}=\boldsymbol{\Lambda}_{i}+t_{i}^{k} \boldsymbol{\Delta}_{\boldsymbol{\Lambda}_{i}}$.

5) Repeat steps 2, 3 and 4 until $\left\|\nabla J\left(\boldsymbol{\Lambda}_{i}\right)\right\|_{2}<\epsilon$, where $\epsilon$ is a small tolerance.

Similarly to [31, Sec. VI], (44) is solved by making use of a basis $\left\{\mathbf{B}_{1}, \mathbf{B}_{2}, \cdots, \mathbf{B}_{N}\right\}$ that span the space of $2 L \times 2 L$ symmetric matrices. More precisely, the procedure is the following

1) Compute

$$
\mathbf{Z}=\int\left(\mathbf{S}_{\tilde{h}}^{-1}(\omega)+\mathbf{\Lambda}_{i}\right)^{-1} d \omega-\mathbf{\Sigma}
$$

2) For each $\mathbf{B}_{n}$, compute

$$
\mathbf{Z}_{n}=\int\left(\mathbf{S}_{\tilde{h}}^{-1}(\omega)+\mathbf{\Lambda}_{i}\right)^{-1} \mathbf{B}_{n}\left(\mathbf{S}_{\tilde{h}}^{-1}(\omega)+\mathbf{\Lambda}_{i}\right)^{-1} d \omega
$$

3) Using the Moore-Penrose pseudoinverse, find $\left\{\mu_{n}\right\}$ satisfying $\mathbf{Z}=\sum_{n} \mu_{n} \mathbf{Z}_{n}$.

4) Set $\boldsymbol{\Delta}_{\boldsymbol{\Lambda}_{i}}=\sum_{n} \mu_{n} \mathbf{B}_{n}$.

The speed of convergence of the optimization procedure strongly depends on the level of similarity between the user-defined covariance matrix $\Sigma$ and the actual covariance matrix of the sea-probed channel. For the simulations conducted in Sec. IV-B and with $\epsilon$ set to $10^{-6}$, it converged in 25 iterations for $\rho_{0}=0.25$ and 200 iterations for $\rho_{0}=0.95$. When the number $L$ of taps becomes large, most of the processing time is consumed by the computation of (47) because the size of the basis $\left\{\mathbf{B}_{1}, \mathbf{B}_{2}, \cdots, \mathbf{B}_{N}\right\}$ also becomes large since $N=L(2 L+1)$.

\section{REFERENCES}

[1] F-X. Socheleau, C. Laot, and J.-M. Passerieux, "A parametric replay-based framework for underwater acoustic communication channel simulation," in Proc. Underwater Communications and Networking (UComms), 2014.

[2] R. Galvin and R.E.W. Coats, “A stochastic underwater acoustic channel model,” in Proc. IEEE Oceans Conf., Sep. 1996. 
[3] P. van Walree, P. Jenserud, and M. Smedsrud, “A Discrete-Time Channel Simulator Driven by Measured Scattering Functions,” IEEE J. Sel. Areas Commun., vol. 26, no. 9, pp. 1628-1637, 2008.

[4] F.-X. Socheleau, C. Laot, and J.-M. Passerieux, "Stochastic Replay of non-WSSUS Underwater Acoustic Communication Channels Recorded at Sea," IEEE Trans. Signal Process., vol. 59, no. 10, pp. 4838-4849, 2011.

[5] R. Otnes, P. van Walree, and T. Jenserud, "Validation of Replay-Based Underwater Acoustic Communication Channel Simulation,” IEEE J. Ocean. Eng., vol. 38, no. 4, pp. 689-700, 2013.

[6] J.-M. Passerieux, F.-X. Socheleau, and C. Laot, "On the capacity of the Underwater Acoustic Communication Channel under Realistic Assumptions,", in Proc. IEEE European Wireless, Apr. 2011, pp. 1-6.

[7] F.-X. Socheleau, M. Stojanovic, C. Laot, and J.-M. Passerieux, "Information-Theoretic Analysis of Underwater Acoustic OFDM Systems in Highly Dispersive Channels," Journal of Electrical and Computer Engineering, 2012, Article ID 716720.

[8] J.-M. Passerieux, F.-X. Socheleau, and C. Laot, "Achievable rates over doubly selective rician-fading channels under peak-power constraint,” IEEE Trans. Wireless Commun., vol. 12, no. 2, pp. 586-594, February 2013.

[9] J.C. Peterson and M.B. Porter, "Ray/beam tracing for modeling the effects of ocean and platform dynamics," IEEE J. Ocean. Eng., vol. 38, no. 4, pp. 655-665, Oct 2013.

[10] X. Cristol, "NARCISSUS-2005: A Global Model of Fading Channel for Application to Acoustic Communication in Marine Environment," in Proc. IEEE Oceans Conf., Brest, Jun. 2005, pp. 655-662.

[11] X. Geng and A. Zielinski, “An eigenpath underwater acoustic communication channel model," in Proc. IEEE Oceans Conf., Oct. 1995.

[12] M. Chitre, "A high-frequency warm shallow water acoustic communications channel model and measurements," J. Acoust. Soc. Am., vol. 122, no. 1, 2007.

[13] F.-X. Socheleau, C. Laot, and J.-M. Passerieux, "A Maximum Entropy Framework for Statistical Modeling of Underwater Acoustic Communication Channels," in Proc. IEEE Oceans'10, May. 2010.

[14] P. Qarabaqi and M. Stojanovic, "Statistical characterization and computationally efficient modeling of a class of underwater acoustic communication channels," IEEE J. Ocean. Eng., vol. 38, no. 4, pp. 701-717, Oct 2013.

[15] H.S. Dol, M.E.G.D. Colin, M.A. Ainslie, P.A. van Walree, and J. Janmaat, "Simulation of an underwater acoustic communication channel characterized by wind-generated surface waves and bubbles," IEEE J. Ocean. Eng., vol. 38, no. 4, pp. 642-654, Oct 2013.

[16] F.-X. Socheleau, J.-M. Passerieux, and C. Laot, "Acoustic Modems Performance Assessment via Stochastic Replay of at Sea Recorded underwater Acoustic Communication Channels," in Proc. Underwater Acoustic Measurement: Technologies and Results, Jun. 2011.

[17] P. van Walree, "Channel sounding for acoustic communications: techniques and shallow-water examples," Research report, Norwegian Defence Research Establishment (FFI), 2011.

[18] R. Otnes, P.A. van Walree, and T. Jenserud, "Erratum to validation of replay-based underwater acoustic communication channel simulation,” IEEE J. Ocean. Eng., vol. 38, no. 4, pp. 809-809, Oct 2013.

[19] F.-X. Socheleau, J.-M. Passerieux, and C. Laot, "Characterisation of Time-Varying Underwater Acoustic Communication Channel with Application to Channel Capacity,” in Proc. Underwater Acoustic Measurement, Jun. 2009.

[20] K. Anim-Appiah, “Complex envelope correlations for non-isotropic scattering," Electronics Letters, vol. 34, no. 9, pp. 918-919, Apr 1998.

[21] C.A. Gutiérrez-Díaz de León and M. Pätzold, "Efficient sum-of-sinusoids-based simulation of mobile fading channels 
with asymmetrical doppler power spectra," in Wireless Communication Systems, 2007. ISWCS 2007. 4th International Symposium on, Oct 2007, pp. 246-251.

[22] G. Deodatis, "Simulation of Ergodic Multivariate Stochastic Processes," Journal of Engineering Mechanics, vol. 122, no. 8, pp. 778-787, 1996.

[23] L. Chen and C. W. Letchford, "Simulation of Multivariate Stationary Gaussian Stochastic Processes: Hybrid Spectral Representation and Proper Orthogonal Decomposition Approach,” Journal of Engineering Mechanics, vol. 131, no. 8, pp. 801-808, 2005.

[24] Q. Ding, L. Zhu, and H. Xiang, "An efficient ergodic simulation of multivariate stochastic processes with spectral representation," Probabilistic Engineering Mechanics, vol. 26, no. 2, pp. 350 - 356, 2011.

[25] E.T. Jaynes, "Information Theory and Statistical Mechanics," Physical review, vol. 106, no. 4, pp. 620-630, 1957.

[26] J. Shore and R. Johnson, "Axiomatic Derivation of the Principle of Maximum Entropy and the Principle of Minimum Cross-Entropy," IEEE Trans. Inf. Theory, vol. 26, no. 1, pp. 26-37, 1980.

[27] J. P. Burg, Maximum Entropy Spectral Analysis, Ph.D. thesis, Department of Geophysics, Stanford University, 1975.

[28] M. Debbah and R. R. Muller, "MIMO Channel Modeling and the Principle of Maximum Entropy," IEEE Trans. Inf. Theory, vol. 51, no. 5, pp. 1667-1690, 2005.

[29] F.-X. Socheleau, C. Laot, and J.-M. Passerieux, "Concise Derivation of Scattering Function from Channel Entropy Maximization,” IEEE Trans. Commun, vol. 58, no. 11, Nov. 2010.

[30] A. Ferrante, C. Masiero, and M. Pavon, "Time and spectral domain relative entropy: A new approach to multivariate spectral estimation," IEEE Trans. Autom. Control, vol. 57, no. 10, pp. 2561-2575, Oct 2012.

[31] A. Ferrante, M. Pavon, and M. Zorzi, "A maximum entropy enhancement for a family of high-resolution spectral estimators," Automatic Control, IEEE Transactions on, vol. 57, no. 2, pp. 318-329, Feb 2012.

[32] T. Cover and J. Thomas, Elements of Information Theory, Wiley, 1991.

[33] M. Pätzold, Mobile fading channels, Wiley, 2002.

[34] J.R. Magnus and H. Neudecker, Matrix Differential Calculus with Applications in Statistics and Econometrics, John Wiley, Chichester, 1999.

[35] G. Matz, “On Non-WSSUS Wireless Fading Channels,” IEEE Trans. on Wireless Commun., vol. 4, no. 5, 2005.

[36] P.A. van Walree, "Propagation and scattering effects in underwater acoustic communication channels," IEEE J. Ocean. Eng., vol. 38, no. 4, pp. 614-631, Oct 2013.

[37] S. H. Huang, T. C. Yang, and Chen-Fen Huang, "Multipath correlations in underwater acoustic communication channels," The Journal of the Acoustical Society of America, vol. 133, no. 4, pp. 2180-2190, 2013.

[38] F. D. Neeser and J. L. Massey, "Proper Complex Random Processes with Applications to Information Theory ," IEEE Trans. Inf. Theory, vol. 39, no. 4, pp. 1293-1302, 1993.

[39] M. Stojanovic, J. A. Catipovic, and J. G. Proakis, "Phase-coherent digital communications for underwater acoustic channels," IEEE J. Ocean. Eng., vol. 19, no. 1, 1994.

[40] J. Nocedal and S. J. Wright, Numerical optimization, Springer, Berlin, 1999. 
Francois-Xavier Socheleau (S'08-M'12) graduated in electrical engineering from ESEO, Angers, France, in 2001 and received the Ph.D. degree from Telecom Bretagne, Brest, France, in 2011.

PLACE

From 2001 to 2004, he was a Research Engineer at Thales Communications, France, where he worked on PHOTO

HERE electronic warfare systems. From 2005 to 2007, he was employed at Navman Wireless (New Zealand/U.K.) as an R\&D Engineer. From 2008 to 2011, he worked for Thales Underwater Systems, France. In November 2011, he joined ENSTA Bretagne as an Assistant Professor. Since September 2014, he has been an Associate Professor at Telecom Bretagne, France. His research interests lie in the field of signal processing and underwater acoustics.

Christophe Laot (M'07-SM'12) was born in Brest, France, on March 12, 1967. He received the Eng.

PLACE degree from the Ecole FRancaise d'Electronique et d'Informatique (EFREI), Paris, France, in 1991 and

PHOTO

HERE the Ph.D degree from the University of Rennes, France, in 1997.

In 1997, he joined the Signal and Communications department, TELECOM Bretagne, Brest, France, as an Associate Professor. Since 2013, he has been full Professor in the same institution. His research interests lie in the areas of communications and signal processing, including equalization, turbo-equalization, iterative receivers for interference cancellation, synchronization and underwater acoustic communications. Dr C. Laot is member of the IEEE communication society and vice-chair (Europe) of the technology committee Underwater Communication, Navigation and Positioning for the IEEE Oceanic Engineering Society.

\begin{tabular}{|c|}
\hline \\
PLACE \\
PHOTO \\
HERE \\
\hline
\end{tabular}

Jean-Michel Passerieux BLABLABLA 